\title{
EVALUATION OF LANE DEPARTURE WARNINGS FOR DROWSY DRIVERS
}

\author{
Ksenia Kozak, Jochen Pohl, Wolfgang Birk, Jeff Greenberg, Bruce Artz, Mike Blommer, Larry Cathey, \\ Reates Curry \\ Ford Motor Company, Dearborn, MI
}

\begin{abstract}
Lane departure warning (LDW) is a driver warning system designed to reduce the number of unintended lane departures. We addressed warning effectiveness and customer acceptance when the unintended lane departures are the result of drowsy driving. Thirty-two adults who were sleep deprived for 23 hours participated in the study and drove Ford's VIRTTEX driving simulator. Four Human Machine Interfaces (HMI) for LDW were evaluated: Steering Wheel Torque, Rumble Strip Sound, Steering Wheel Vibration and Head Up Display. A yaw deviation technique was used to produce controlled lane departures in the first two hours of the drive while for the last 20 minutes driver-initiated lane departures were analyzed. The Steering Wheel Vibration HMI, accompanied by Steering Wheel Torque, was found to be the most effective HMI for LDW in a group of drowsy drivers, with faster reaction times and smaller lane excursions. The Vibration HMI was also perceived by the drowsy drivers to be acceptable and helpful.
\end{abstract}

\section{INTRODUCTION}

Driver warning systems are designed to help reduce the exposure of occupants to crashes by helping the driver preemptively avoid them. Lane departure warning (LDW) is a driver warning system designed to help the driver reduce the number of unintended lane departures. Many single vehicle roadway departure crashes take place in light traffic situations and good weather conditions. Such crashes are often due to drivers' inattention or drowsiness. Drowsiness is frequently reported during night-time driving and in monotonous driving conditions (Horne \& Reyner, 1995), with 51\% of drivers reporting that they have driven a vehicle while feeling drowsy in the past year. NHTSA data (Royal, 2002) indicates that in recent years there have been about 100,000 crashes annually in which police cited driver drowsiness, resulting in about 1,500 fatalities.

The goal of a LDW is to warn drivers if they are unintentionally drifting out of their lanes (Figure 1). The system will not take any automatic action to prevent a possible lane departure. Responsibility for the safe operation of the vehicle remains with the driver. LDW relies on the detection of the vehicle position with respect to the road lane markings in order to detect a lane departure. The vehicle position is evaluated by a camera system mounted behind the windshield. The principal system layout is illustrated in Figure 2.

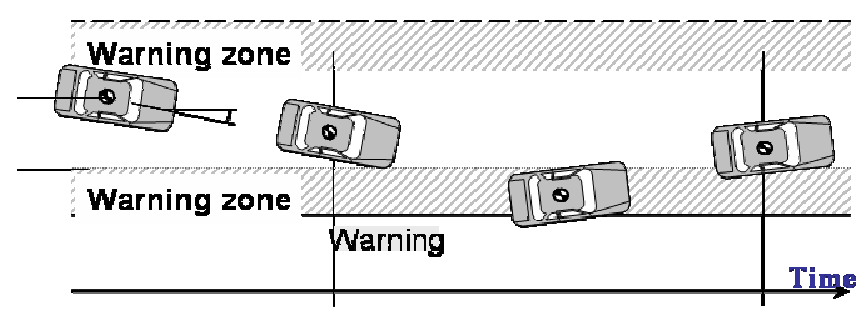

Figure 1. Vehicle drifting out of lane and receiving a LDW.

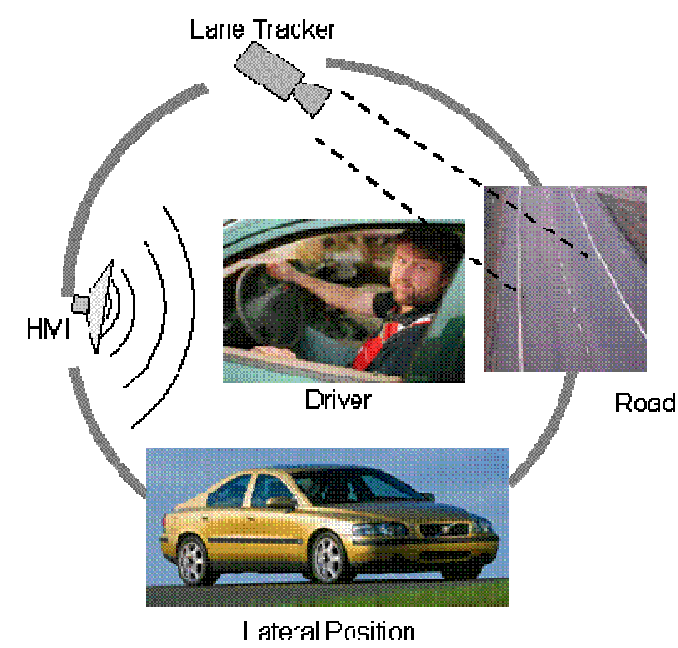

Figure 2. Basic principle of operation and system components for LDW

Lane tracking performance is dependent on the quality of lane markings, lighting conditions and environmental factors such as rain, snow and fog which reduce visibility. The driver interface must be matched to the overall system performance so that the warning is effective, but does not lead to driver annoyance due to false warnings. This study was designed to address warning effectiveness and customer acceptance when the unintended lane departures are the result of drowsy driving.

\section{METHODS}

The study was conducted in Ford's VIRtual Test Track Experiment (VIRTTEX), a hydraulically powered, 6degrees-of-freedom moving base driving simulator, with a 2000 Volvo S80 as the test vehicle. The drive took place on a 
simulated section of a US interstate during night-time conditions. Drivers were instructed to drive in the right lane only, which had a lane width of $12 \mathrm{ft}$. The shoulders of the road had increased road roughness to simulate actual road conditions during a lane departure. The road consisted of two lanes in each direction. Fast-moving, overtaking traffic was present in the left lane, while opposing traffic did not interact with the driver. Traffic density was very light.

\section{Subjects}

Thirty-two drivers participated in the study. Subjects were licensed drivers who consumed no more than an average amount of caffeine (500mg or less, approximately 5 cups of coffee), had a stable sleep/wake cycle and were free of sleep disorders. Subjects did not sleep the night before the simulator drive and were not allowed to have caffeine after 6:00 pm the day before the drive. When their drive began, subjects had been awake for about 23 hours. The amount of sleep was verified by a wrist-actimeter. Actimeters are used to measure fine limb movements, usually of the wrist, which are indicative of sleep disturbance. Actimeters are small devices worn like a wristwatch and easily used in the home without supervision. They log and store data for many nights, which is later transferred to a computer for conversion to actigrams, the graphical records of limb movements.

\section{Drowsiness Measures}

Drowsiness was assessed using three measures: a physiological measure of eye closure (PERCLOS), a sustained reaction time task based on the Psychomotor Vigilance Task and a subjective assessment using the Karolinska Sleepiness Scale (KSS). The sustained reaction time task and KSS have been described previously (Kozak et al., 2005).

PERCLOS is a measure of drowsiness that is characterized by slow ramp closure of the eye and is defined as the proportion of time that the eyes are $80-100 \%$ closed over a specified interval, often one minute (Wierwille et al., 1994). An ISCAN Eye Tracker, mounted on a baseball cap, was used to obtain a high resolution eye image for the calculation of PERCLOS. A PERCLOS value of $8 \%$ was used to define a drowsy driver (Grace and Steward, 2003).

\section{Lane Departure Warnings}

Four Human Machine Interfaces (HMI) for LDW were evaluated. Only the Steering Wheel Torque HMI was presented alone. The Rumble Strip Sound, Steering Wheel Vibration and Head Up Display HMIs were always accompanied by the Steering Wheel Torque HMI.

- $\quad$ Steering Wheel Torque. The Steering Wheel Torque was designed to communicate to the driver the appropriate steering wheel angle required in order to return to the lane. Inputs to the algorithm were distances to the right and left lane markings. The lateral velocity components were used to provide lateral damping to avoid a pendulum motion between the lines. The Torque HMI was presented alone and it accompanied the warnings described below.

- $\quad$ Rumble Strip Sound with Steering Wheel Torque. The synthesized Rumble Strip Sound was designed based on recordings from a vehicle driving over gapped rumble strips at $65 \mathrm{mph}$ on an interstate highway (Figure 3).

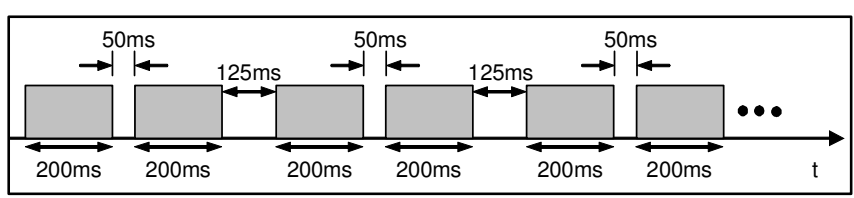

Figure 3. Timing of the Rumble Strip Sound warning

- $\quad$ Steering Wheel Vibration and Steering Wheel Torque. The Steering Wheel Vibration was a $15 \mathrm{~Hz}$ vibration with $2 \mathrm{Nm}$ peak amplitude and duration of $1.5 \mathrm{~s}$.

- Head Up Display (HUD) and Steering Wheel Torque. The HUD was a row of flashing red LEDs, mounted on top of the instrument panel and reflected into the driver's field of view via the windshield (Figure 4).

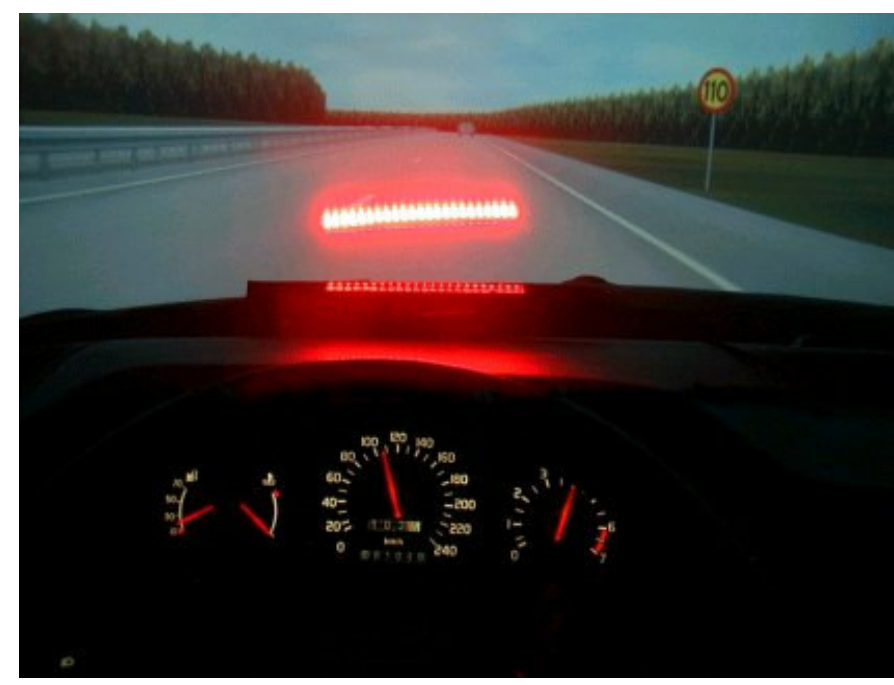

Figure 4. HUD warning reflected on the windshield

\section{Experimental Design}

Upon arriving at the VIRTTEX Lab at 5:30 AM, the subjects watched a safety video, signed a consent form and were provided initial training with a computer presentation. At 6:00 AM the subjects were escorted into the simulator and began the simulated drive. Within the first 5 minutes of the drive the subjects experienced each of the four LDW HMIs for training purposes. The subjects rated their sleepiness level and 
completed three sustained reaction time tasks at 15, 75 and 135 minutes into the drive. For the first two hours of the drive, operator-initiated yaw deviations were used to produce controlled lane departures at random intervals between 5 and 15 minutes. During these forced lane departures, drivers experienced the four HMIs or no warning in a randomly determined order. Each HMI and the null condition were experienced twice. For the final 20 minutes of the drive, the LDW HMIs were activated in a random order only for driver-initiated lane departures. After each lane departure drivers were asked if they noticed a warning. If they did, drivers were then asked to assess the warning based on their perception of its helpfulness, intuitiveness, acceptability and whether they would like this system in their vehicle. Driving performance was assessed using measures of reaction time to warning, magnitude of lane departure and time spent outside the lane. The length of the drive was approximately three hours.

\section{Controlled Lane Departures}

A unique motion control strategy was developed to produce a controlled lane departure in order to compare mitigation strategies. The technique allowed each driver to experience the same rate of lane departure. The controlled lane departure was implemented by adding a small yaw deviation sequence to the vehicle dynamics model. This modified vehicle dynamics information was sent to everything except the motion control algorithm. The driver appeared to be departing the lane visually, yet the driver did not experience any perceptible motion cues from the yawing.

Figure 5 shows the $\sin ^{2}$ profile that was used in the study to generate 5 degrees of yaw over 5 seconds. During the study, the direction of the yaw deviation was automatically determined by the driver's lane position at the time the yaw deviation was initiated; if the lane position was closer to the right shoulder or left lane, then the yaw deviation was to the right or left, respectively.

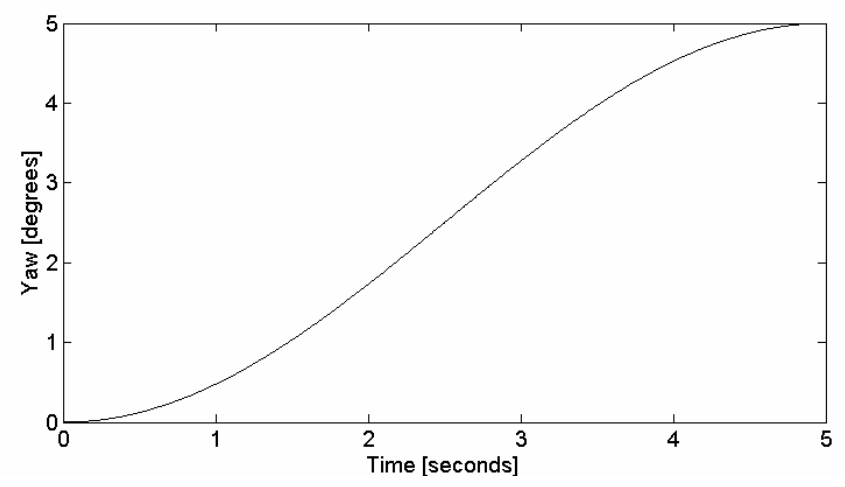

Figure 5. Profile of a yaw deviation to the right (yaw is defined as positive in a clock-wise direction)

\section{Driving Performance Measures for LDWs}

Reaction time to $L D W$. The time it took drivers to react to a warning was inferred from steering wheel movement. The time at which the driver reacted to the warning was defined by first locating the maximum steering wheel velocity (when steering correction reaches maximum velocity) between the start of the yaw deviation and the maximum lane excursion for controlled lane departures or between the beginning of the lane departure and the maximum lane excursion for subject initiated lane departures. Looking back in time, the first steering wheel reversal was identified. Within the interval defined by the steering wheel reversal and maximum steering wheel velocity, the maximum steering wheel acceleration was identified. Finally, we looked back in time from the maximum steering wheel acceleration to the first acceleration zero crossing. The reaction time is defined as the time interval from the initiation of the LDW to the acceleration zero crossing (Figure 6).

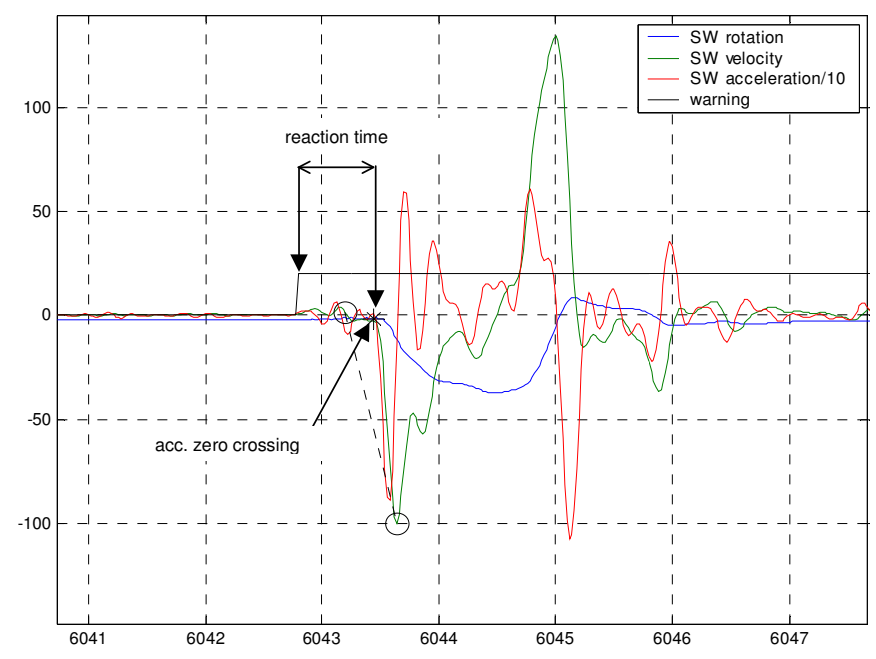

Figure 6. Example of experimental results showing steering wheel rotation, velocity and acceleration and onset of warning for the calculation of reaction time

Lane excursions. A lane excursion was defined as the distance between the outer edge of the lane markings and the outer edge of the leading tire. Several lane departure related measures were computed following a LDW: maximum lane excursion, integrated lane excursion, rms lane excursion and the time spent outside the lane. These measures were used to assess the efficacy of the various HMIs in helping a drowsy driver who has experienced an unintended lane departure return back to his lane. 


\section{RESULTS}

When all lane departures were analyzed, significant differences were not apparent. However, when PERCLOS was used to identify those events where the driver was drowsy, significant differences emerged. For the following analyses we analyzed only those lane departures that occurred when the subject was drowsy, as measured by a PERCLOS value greater than or equal to $8 \%$.

\section{Reaction Time to Warning}

All four HMIs provided improved recognition of an impending lane departure as measured by steering reaction time (RT). When no warning was present, drowsy drivers' RT, calculated from the moment a warning would have been given, ranged from 0.58 to $2.36 \mathrm{~s}$. The presence of a LDW significantly reduced the RT almost in half from a mean of 1.17 to $0.62 \mathrm{~s}$ (ANOVA, $\mathrm{p}<0.05$ ). Post-hoc t-tests indicated that the Steering Wheel Vibration HMI was significantly different from no warning $(\mathrm{p}<0.05)$. RT with a Vibration HMI decreased the average RT to $0.46 \mathrm{~s}$ (Figure 7). The Rumble Strip Sound HMI decreased the average RT to $0.55 \mathrm{~s}$, a result that approached significance $(\mathrm{p}=0.06)$. Both Vibration and Rumble Strip Sound HMI produced consistent results between subjects as indicated by small variances and tight distributions.

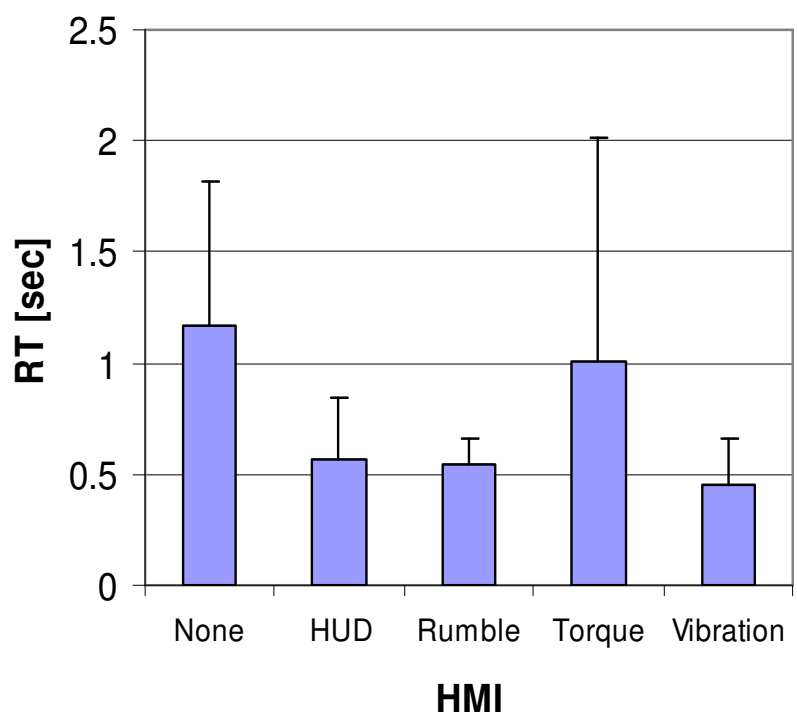

Figure 7. Average steering wheel reaction time for each warning

\section{Lane Excursions}

The integrated lane excursion was significantly smaller (ANOVA, $\mathrm{p}<0.05)$ when a LDW was present. The integrated lane excursion decreased from an average value of $4.44 \mathrm{ft}-\mathrm{s}$ when no warning was given to an average of $2.44 \mathrm{ft}-\mathrm{s}$ when a LDW was present. Post-hoc t-tests indicated that the Steering Wheel Vibration HMI was significantly different from no warning $(\mathrm{p}<0.05)$ with an average integrated lane excursion of $1.39 \mathrm{ft}-\mathrm{s}$. The maximum lane excursion, the rms of the lane excursion and the total time outside of the lane did not significantly differ when a LDW was present.

\section{Subjective Assessment}

The Vibration and the Rumble Strip HMIs received higher average helpfulness and acceptability scores than the HUD and Torque Ony HMIs ( $<<0.05)$. Drivers noticed only $32 \%$ of all Torque warnings, a rate similar to the $33 \%$ of drivers who said they noticed a warning when none was actually given. All other HMIs were noticed at least $98 \%$ of the time and this was significantly different from the Torque Only HMI $(\mathrm{p}<0.05)$.

\section{DISCUSSION}

The Steering Wheel Vibration, accompanied by the Steering Wheel Torque, was found to be the most effective HMI for LDW in a group of drowsy drivers. The Vibration HMI improved the driving performance of drowsy drivers during a lane departure, resulting in a faster reaction time following a warning and a decrease in the magnitude of the lane excursion. The Vibration HMI not only improved driving performance, it was also acceptable to the drowsy drivers and perceived as helpful. The advantages of the Rumble Strip Sound HMI were also apparent in this study. The Rumble Strip Sound HMI exhibited a trend for decreasing the reaction time following a warning and was well liked by drowsy drivers.

Both the Vibration and the Rumble Strip Sound HMIs mimic the warming drivers get when they drive over a rumble strip on the shoulder of a road. Rumble strips have been shown to reduce single vehicle roadway departure crashes by 30-50\% (Jeftic et al., 2003). This similarity adds scenaric transparency to these warnings increasing the chances that the driver will understand and heed the warning.

Several factors were instrumental in the success of this study. First, by using PERCLOS to quantify the degree of drowsiness, we assessed the efficacy and acceptance of a LDW at specific times when a driver was drowsy. Second, the yaw deviation was successfully implemented as a means to create a controlled, uniform lane departure for all subjects. The subjects' comments suggested that they perceived the yaw deviations as self-initiated lane departures. Finally, the three hour duration increased drowsiness with time-on-task and allowed sufficient time for the drivers to potentially experience each HMI three times. 
By assessing the state of the driver, their driving performance and their perceptions, this study has contributed towards knowledge of how to design a LDW system for drowsy drivers. However, more work needs to be done to investigate the issue of behavioral adaptations to LDW systems. This study does not address whether a LDW system may lead to drowsy drivers driving longer and in a drowsier state than they would without such a system.

\section{REFERENCES}

Grace, R., \& Steward, S. (2003). Drowsy driver monitor and warning system. In Proceedings of the 2003 Driving Assessment Conference.

Horne, J.A., \& Reyner, L.A. (1995). Sleep related vehicle accidents. British Medical Journal, 310, 565-567.

Jeftic, Z., Engström, J. \& Piamonte, P. (2003). Potential safety benefits of lane departure warning systems on Swedish roads-prestudy. Vagverket publication 2003:14. Volvo Technology Corporation.
Kozak, K., Curry, R., Greenberg, J., Artz, B., Blommer, M., \& Cathey, L. (2005). Leading indicators of drowsiness in simulated driving. In Proceedings of the Human Factors and Ergonomics Society $49^{\text {th }}$ Annual Meeting. Santa Monica, CA: Human Factors and Ergonomics Society.

Royal, D. (2002). National Survey of Distracted and Drowsy Driving Attitudes and Behavior: 2002 (DOT HS 809 566). Washington, D.C.: National Highway Traffic Safety Administration.

Wierwille, W.W., Ellsworth, L.A., Wreggit, S.S., Fairbanks, R.J., \& Kirn, C.L. (1994). Research on vehicle-based driver status/performance monitoring: development, validation, and refinement of algorithms for detection of driver drowsiness. (DOT HS 808 247). Washington, D.C.: National Highway Traffic Safety Administration. 